\title{
Characterization of Focal Liver Lesions Indistinctive on B Mode Ultrasound: Benefits of Contrast-Enhanced Ultrasound
}

\author{
Yi Dong, ${ }^{1}$ Feng Mao, ${ }^{2}$ Jiaying Cao, ${ }^{1}$ Peili Fan, ${ }^{2}$ and Wen-Ping Wang ${ }^{1}$ \\ ${ }^{1}$ Department of Ultrasound, Zhongshan Hospital, Fudan University, Shanghai 200032, China \\ ${ }^{2}$ Shanghai Institute of Imaging, 180 Fenglin Road, Shanghai 200032, China \\ Correspondence should be addressed to Wen-Ping Wang; puguang61@126.com
}

Received 6 February 2017; Accepted 30 March 2017; Published 11 April 2017

Academic Editor: Fumio Imazeki

Copyright (C) 2017 Yi Dong et al. This is an open access article distributed under the Creative Commons Attribution License, which permits unrestricted use, distribution, and reproduction in any medium, provided the original work is properly cited.

Aim. The aim of this prospective study was to evaluate the additional value of contrast-enhanced ultrasound (CEUS) in identifying and characterizing of focal liver lesions (FLLs) that are indistinctive on B mode ultrasound (BMUS). Methods. The study focused on 70 consecutive patients (male 46, female 24; mean age, 53.1 years \pm 10 ). All lesions were detected by MRI but could not be clearly visualized by BMUS. CEUS was performed by injected SonoVue ${ }^{\circledR}$ (Bracco Imaging Spa, Milan, Italy) as a quick bolus into the antecubital vein. All lesions were proved by pathologic and MRI findings as primary or metastatic hepatic malignancies. Results. On CEUS, 45 (64.2\%) FLLs displayed arterial hyperenhancement and 55 (78.5\%) lesions showed hypoenhancement in portal venous and late phase (PVLP). Homogeneous and complete hyperenhancement pattern during the arterial phase is highly suspicious for HCC in liver cirrhosis (96.8\%). Arterial isoenhancement and early washout during PVLP are characteristic for metastasis (73.3\%). For recurrence lesions, arterial hyperenhancement and isoenhancement during PVLP are more common (60\%). Conclusion. CEUS may provide added diagnostic values in FLLs appearing indistinctive on BMUS. Presence of early arterial enhancement and washout during PVLP may be helpful for detection of those lesions.

\section{Introduction}

Incidentally detected focal liver lesions (FLLs) are more commonly encountered in daily abdominal imaging practice, which may need further clinical investigations [1]. Once a FLL is detected, it is crucial to characterize it with the aim to confirm or rule out HCC or other malignancies [2].

$\mathrm{B}$ mode ultrasound (BMUS) is the most popular used diagnostic tool available for the assessment of FLLs. Therefore, a common practice is to use targeted BMUS immediately to assess the indeterminate small lesion found with CT or MRI. Otherwise, further percutaneous biopsy or radiofrequency ablation (RFA) will always be guided by realtime BMUS. Short-term BMUS follow-up may be needed to further characterize the lesion [3]. Previous study showed the detection and characterization ability of BMUS is higher if the lesion detected with CT is $>5 \mathrm{~mm}$ [3]. However, BMUS still shows low specificity and low accuracy for optimal characterization of small FLLs $[4,5]$. Detection and differentiation of benign or malignant FLLs on a significant cirrhotic background can be a challenge [6,7]. Detection of isoechoic or small (less than $1 \mathrm{~cm}$ in diameter) FLLs is also difficult with BMUS [8]. As reported in the literature, some metastases are still undetected in BMUS, which is currently the benchmark staging method in patients with colon cancer [9].

In recent years, contrast-enhanced ultrasound (CEUS) has demonstrated a significant efficacy and dramatic improvement in either detection or characterization of FLLs. Previously reported data for the differentiation of FLLs showed good accuracies for CEUS, ranging from $85 \%$ to $91 \%$, with moderate interobserver agreement [10]. CEUS enjoyed a real-time diagnostic accuracy similar to that of computed tomography (CT) and magnetic resonance (MR) imaging, without the use of ionizing radiation or nephrotoxic agents [11-14]. CEUS was also proved to be possible to achieve a significant improvement in sensitivity for metastasis detection or excluding metastases in cancer patients with MDCT evidence of subcentimetric, indeterminate focal liver lesions [3], which is also comparable to that of CT or MRI [15-17]. 
The European Federation of Societies for Ultrasound in Medicine and Biology (EFSUMB) guidelines and comments of the guidelines recommend the use of CEUS to diagnose suspected lesions identified in a background of chronic hepatitis or liver cirrhosis, also in patients with a known history of malignancy $[18,19]$. CEUS is discussed for the recall and characterization of FLLs $\geq 1 \mathrm{~cm}$ based on contrast-enhanced imaging techniques with the use of vascular contrast media [2]. However, only a few studies have been conducted using CEUS to determine the enhancement pattern of those atypical or indistinctive lesions on BMUS.

The purpose of our current prospective study was to assess the additional value of the CEUS in identification and characterization of those histologically confirmed FLLs which were indistinctive or undetermined on BMUS.

\section{Materials and Methods}

2.1. Institutional Board Approval. This prospective study was approved by our institutional review board. All patients gave their full informed consent before the CEUS examination. The procedure followed was in accordance with the Declaration of Helsinki.

2.2. Patients. Between Feb 2012 and June 2016, 1250 patients were referred to our institution for liver CEUS assessment. Among them, 70 consecutive patients (24 women and 46 men; age range: $22-84$ years, mean: 55 years \pm 13 ) were detected by MRI but could not be clearly visualized by BMUS (Table 1). The patients' inclusions criteria were as follows: all lesions were detected as malignant FLLs by MRI in the last month; patients need further ultrasound guided biopsy or minimal invasive treatment (such as RFA); lesions were nonvisible on BMUS ultrasound during the regular clinical procedure. The exclusion criteria were age $<18$ year, recent cardiac infarct, and known allergic reactions to CEUS contrast agents.

The final diagnoses for 43 patients were based on histopathologic results obtained from ultrasound guided 18gauge core-needle biopsy $(n=6)$ or surgery $(n=37)$. For the remaining 27 patients without histologic confirmation, typical appearance of these lesions on contrast-enhanced MR images, with systematic follow-up (at least 12 months), was considered the reference standard.

2.3. Examination Technique. Two experienced radiologists (more than 15 years' experience in CEUS of the liver), who were aware of the patients' clinical histories, performed ultrasound scanning with a Siemens S2000 ultrasound system (Siemens AG, Erlangen, Germany, 4C-1 transducer) or LOGIQ E9 ultrasound system (GE Healthcare, Milwaukee, WI, USA, C1-5-D transducer).

A baseline ultrasound examination, including grey scale and color flow imaging analysis, was performed. After detailed evaluation of prior MR images, BMUS was used to examine the whole liver and searched for the suspected FLL. Intrahepatic anatomic structures such as cysts, blood vessels, gallbladder, or scars were used as references. Optimized instrument settings were used to acquire more clear
TABLE 1: Baseline characteristics of patients.

\begin{tabular}{lc}
\hline Characteristic & $\begin{array}{c}\text { Patients } \\
(n=70)\end{array}$ \\
\hline Age (year) & $55 \pm 13$ \\
$\quad$ Mean \pm SD & $22-84$ \\
Range & $46 / 24$ \\
Male/female & \\
Underlying liver diseases & $27(25 / 2)$ \\
Cirrhosis (viral/alcohol) & $26(25 / 1)$ \\
Fibrosis (viral/alcohol) & 15 \\
Previous tumor history & 2 \\
None & \\
AFP (ng/mL) & $46(65.7 \%)$ \\
$\leq 20, n(\%)$ & $17(24.3 \%)$ \\
$21-200, n(\%)$ & $7(10 \%)$ \\
$>200, n(\%)$ & \\
CA 19-9 ( $\mu / \mathrm{mL})$ & $6(8.5 \%)$ \\
$\leq 4.9, n(\%)$ & $64(91.5 \%)$ \\
$>4.9, n(\%)$ & 37 \\
Final diagnosis & 6 \\
Liver surgery & 27 \\
Core needle biopsy & \\
MR images follow-up & \\
\hline
\end{tabular}

AFP: alpha-fetoprotein; CA19-9: carbohydrate antigen 19-9; HCC: hepatocellular carcinoma; $n$ : number.

visualization and to find the proper location of lesions, such as the adjustment of focal zones, field of view, dynamic range, and application of harmonic imaging. If the FLL was not visible or could not be differentiated from regenerative or cirrhosis nodules, it was regarded to be indistinctive.

A 2-step strategy with repeated injection of SonoVue was applied in all 70 patients. First targeted CEUS were performed to detect any hypoenhanced lesion during portal venous or late phase (PVLP), with SonoVue (Bracco, Milan, Italy) as contrast agent, which was injected intravenously as a $2.4 \mathrm{~mL}$ bolus followed by $5 \mathrm{~mL}$ of normal sterile saline flush via a 22gauge peripheral intravenous cannula. Low mechanical index (MI) ranging from 0.05 to 0.08 was used for real-time CEUS imaging. Each examination lasted for at least 5 minute after bolus injection. Then a further $2.4 \mathrm{~mL}$ bolus of SonoVue was administered to focus on the detailed contrast enhancements of FLLs detected, with an interval time of at least 15 minutes to allow for clearance of the previous injected contrast agents.

Digital cineloops were digitally stored as raw-data in a PC-based workstation connected to the ultrasound equipment.

2.4. Image Analysis. Before CEUS, grey scale echogenicity of the lesions was observed in comparison with adjacent liver parenchyma. We classified those FLL as being minor hyperechoic, isoechoic, and minor hypoechoic.

Immediately after the injection of CEUS agents, 2 examiners evaluated by consensus the dynamic enhancement pattern of each lesion in comparison to surrounding 
TABLE 2: Baseline characteristics of FLL included.

\begin{tabular}{lc}
\hline Characteristic & $\begin{array}{c}\text { FLL lesions } \\
(n=70)\end{array}$ \\
\hline Diameter $(\mathrm{mm})$ & $14.6 \pm 6$ \\
$\quad$ Mean \pm SD & $6-30$ \\
Range & $63(90 \%)$ \\
$\leq 20 \mathrm{~mm}(\%)$ & $7(10 \%)$ \\
$20-30 \mathrm{~mm}(\%)$ & \\
Location & $18(25.7 \%)$ \\
Left lobe, $n(\%)$ & $41(58.5 \%)$ \\
Right lobe, $n(\%)$ & $11(15.8 \%)$ \\
Left \& right lobe, $n(\%)$ & \\
Lesion number, $n(\%)$ & $52(74.3 \%)$ \\
$n=1$ & $12(17.1 \%)$ \\
$n=2$ & $6(8.6 \%)$ \\
$n>3$ & \\
Final diagnosis, $n(\%)$ & $35(50 \%)$ \\
Primary HCC & $20(28.5 \%)$ \\
Recurrent HCC & $15(21.4 \%)$ \\
Metastasis &
\end{tabular}

FLL: focal liver lesion.

liver parenchyma. The pattern of enhancement throughout the arterial, portal venous, and late phases was observed according to the EFSUMB guideline $[18,19]$. The CEUS enhancement patterns during arterial phase were subjectively classified as (1) diffuse homogeneous (entire lesion enhanced rapidly and uniformly); (2) diffuse inhomogeneous (heterogeneous enhancement of the whole lesion); (3) rimlike (peripheral enhancement). Special attention was paid to presence or absence of early arterial enhancement and to the detection of any PVLP washout area of contrast agents.

Before and after CEUS, a 4-point scale was used to grade detection confidence: (1) distinctive; (2) probably visible; (3) poorly visible; (4) invisible [20].

2.5. Statistical Analysis. Statistical analysis was performed with a computer software package (SPSS, version 15.0, IBM corporation, Armonk, USA). The improvement in diagnostic confidence was assessed by receiver operating characteristic (ROC) curves analysis. For all tests a $P$ value $<0.05$ was considered to indicate a statistically significant difference.

\section{Results}

3.1. Final Diagnosis of FLLs. Consequently, 70 FLLs not visible on BMUS in 70 patients were included in this study (Table 2). Single malignant FLLs were detected in 52 patients and multiple lesions in 18 patients. For multiple FLLs, only the biggest one was evaluated during our current study. Final diagnosis proved 35 primary HCCs, 20 recurrent HCCs, and 15 metastasis malignancies. The median size of those FLLs was $16 \mathrm{~mm}$ (size range: $6-30 \mathrm{~mm}$; mean \pm SD: $14.6 \pm 6 \mathrm{~mm}$ ).

3.2. Detection Rate of FLLs. All those lesions were isoechoic $(n=45)$, slightly hyperechoic $(n=11)$, or slightly hypoechoic
TABLE 3: Detection of FLL not visible on conventional BMUS.

\begin{tabular}{lc}
\hline Characteristic & $\begin{array}{c}\text { FLL lesions } \\
(n=70)\end{array}$ \\
\hline BMUS, $n(\%)$ & $45(45.4 \%)$ \\
Isoechoic & $11(46.8 \%)$ \\
Slightly hyperechoic & $14(7.8 \%)$ \\
Slightly hypoechoic & \\
Detected by first CEUS & $45(64.2 \%)$ \\
Arterial hyperenhancement, $n(\%)$ & $55(78.5 \%)$ \\
PVLP hypoenhancement, $n(\%)$ &
\end{tabular}

FLL: focal liver lesion; BMUS: B mode ultrasound; CEUS: contrast enhanced ultrasound.

TABLE 4: Results of 4-point scale grade of BMUS and CEUS in 70 indistinctive FLLs.

\begin{tabular}{lcc}
\hline Four-point scale of indistinctive lesions & BMUS & CEUS \\
\hline Grade 1, invisible & 8 & 0 \\
Grade 2, poorly visible & 3 & 5 \\
Grade 3, probably visible & 0 & 15 \\
Grade 4, distinctive & 0 & 50 \\
\hline
\end{tabular}

FLL: focal liver lesion; BMUS: B mode ultrasound; CEUS: contrast enhanced ultrasound.

$P=0.008$ (Fisher's exact test).

( $n=14)$ with indistinctive margins on BMUS. After first CEUS procedure, $64.2 \%$ of FLLs showed hyperenhancement during arterial phase and $78.5 \%$ lesions showed hypoenhancement during PVLP (Table 3).

Comparing the 4-point scale classification results between BMUS and CEUS, among 70 FLLs diagnosed on MR imaging, BMUS identified 11 (15.7\%) lesions as poorly or probably visible lesions. CEUS detected an additional 59 $(84.3 \%)$ lesions (Table 4$)$, which was significantly higher than BMUS $(P<0.05)$.

3.3. CEUS Enhancement Pattern (Repeated Injection). After repeated injection of SonoVue, the contrast enhancements of FLLs were evaluated as well focussing on the lesion detected, during the arterial phase (10-30 seconds), portal venous (30-120 seconds), and late phases (120-300 seconds). On CEUS, homogeneous and complete hyperenhancement pattern during the arterial phase is highly suspicious for HCC in liver cirrhosis patients (96.8\%) (Figure 1). Arterial isoenhancement early washout during PVLP is characteristic for metastasis malignancies (54.7\%) (Figure 2). Arterial hyperenhancement and isoenhancement during PVLP are more common for recurrent HCCs (54.7\%) (Figure 3) (Table 5).

\section{Discussion}

According to the EFSUMB guideline, CEUS allowed the characterization of most of FLLs by analysis of the arterial, portal venous, and late phases $[18,19]$. Previously studies reported that CEUS has a high diagnostic accuracy for the differential diagnosis of FLLs based on description of tumorspecific enhancement patterns, obtaining a sensitivity of $90 \%$, a specificity of $99 \%$, and an accuracy of $89 \%$ for the 
TABLE 5: Contrast enhancement features of 70 indistinctive lesions BMUS.

\begin{tabular}{|c|c|c|c|}
\hline Characteristic & $\begin{array}{l}\text { Primary HCCs } \\
\quad(n=35)\end{array}$ & $\begin{array}{l}\text { Recurrence HCCs } \\
\qquad(n=20)\end{array}$ & $\begin{array}{c}\text { Metastasis } \\
(n=15)\end{array}$ \\
\hline \multicolumn{4}{|l|}{ Arterial phase enhancement, $n(\%)$} \\
\hline Hyperenhanced & $26(74.2 \%)$ & $16(80 \%)$ & $3(20 \%)$ \\
\hline Isoenhanced & $9(25.8 \%)$ & $4(20 \%)$ & $12(80 \%)$ \\
\hline \multicolumn{4}{|l|}{ Type of arterial phase enhancement, $n(\%)$} \\
\hline Diffuse homogeneous enhancement & $25(71.4 \%)$ & $12(60 \%)$ & $1(6.7 \%)$ \\
\hline Diffuse inhomogeneous enhancement & $10(28.6 \%)$ & $7(35 \%)$ & $2(13.3 \%)$ \\
\hline Rim-like hyperenhancement & 0 & $1(5 \%)$ & $12(80 \%)$ \\
\hline \multicolumn{4}{|l|}{ Portal venous phase enhancement, $n(\%)$} \\
\hline Isoenhanced & $20(57.1 \%)$ & $15(75 \%)$ & $4(26.7 \%)$ \\
\hline Hypoenhanced & $15(42.9 \%)$ & $5(25 \%)$ & $11(73.3 \%)$ \\
\hline \multicolumn{4}{|l|}{ Late phase enhancement, $n(\%)$} \\
\hline Isoenhanced & $2(5.7 \%)$ & $12(60 \%)$ & $1(6.7 \%)$ \\
\hline Hypoenhanced & $33(94.2 \%)$ & $8(40 \%)$ & $14(93.3 \%)$ \\
\hline
\end{tabular}

BMUS: B mode ultrasound; HCC, hepatocellular carcinoma; CEUS: contrast enhanced ultrasound.

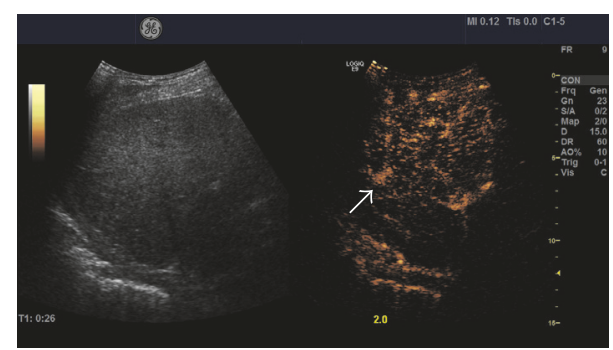

(a)

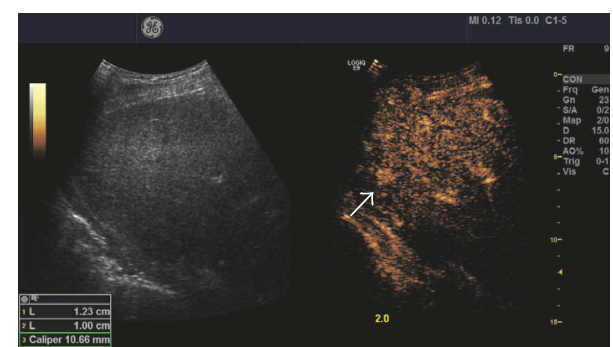

(b)

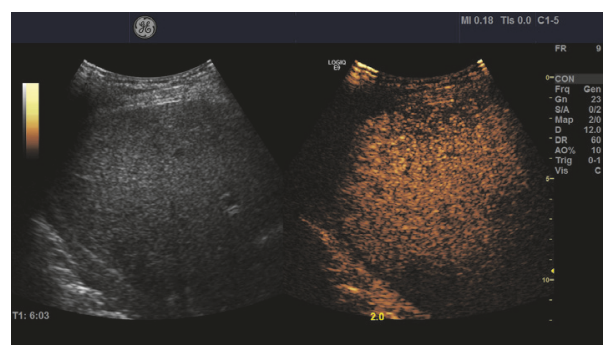

(c)

FIGURE 1: Small HCC (10 mm) nonvisible on B mode ultrasound but was hyperenhanced during arterial phase (a) and portal venous (b) phase. It was slightly hypoenhanced in the late phase (c) on contrast-enhanced ultrasound. The arrow in (a) and (b) refers to the hyperenhanced small HCC lesion.

diagnosis for the discrimination of malignant and benign FLLs when compared with BMUS [21]. Depending on the presence of early arterial enhancement, and by detection of PVLP washout, our results indicated that CEUS may play a confirmatory role in the detection of those indistinctive FLLs on BMUS. Detailed scanning during the late phase of CEUS enables better detection of malignant FLLs [22].

Ultrasound contrast agents are strictly intravascular, without diffusion into the interstitial space. This explains that evidence of washout during PVLP is the most important feature in the differentiation of FLLs $[18,19]$. Also, it allows for discrimination between types of malignancy [14]. In our current study, early and complete washout during PVLP is typical for metastases FLLs. However, HCCs often showed a relatively slower washout and $57.1 \%$ of HCCs still are isoenhanced during portal venous phase.

Echogenicity of FLL depends on its size and on the echo difference compared with surrounding liver parenchyma. BMUS recognition of HCC in liver cirrhosis can be difficult if the echo texture is very inhomogeneous [23]. Although many HCCs demonstrate arterial phase enhancement and moderate washout during PVLP, some small HCCs are seen 


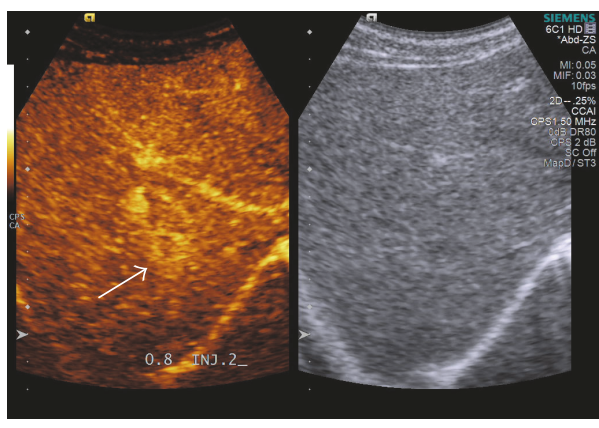

(a)

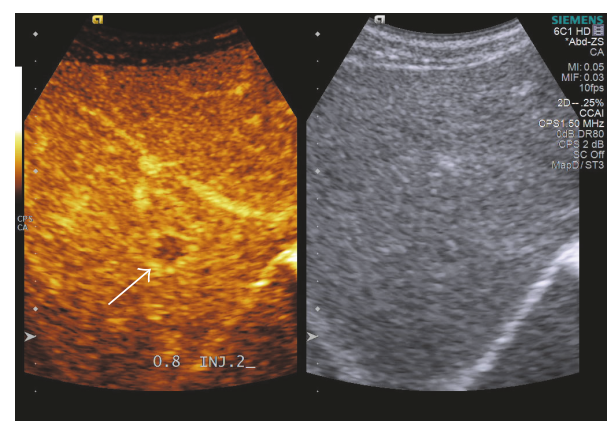

(b)

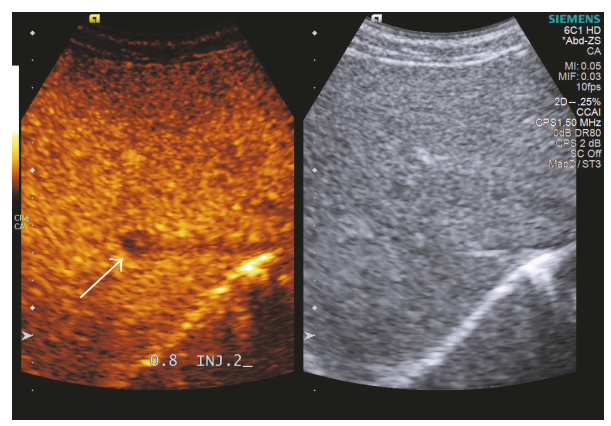

(c)

FiguRE 2: Small metastasis lesion from colon $(15 \mathrm{~mm})$ not clearly visible on B mode ultrasound showed rim-like hyperenhancement during arterial phase (a). Clear hypoenhancement during the portal venous (b) and late phase (c) on contrast-enhanced ultrasound. The arrow in the figure refers to rim-like hyperenhanced small metastasis lesion during arterial phase and showed clear hypoenhancement during the portal venous.

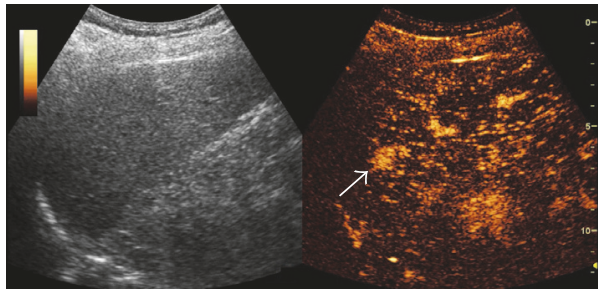

(a)

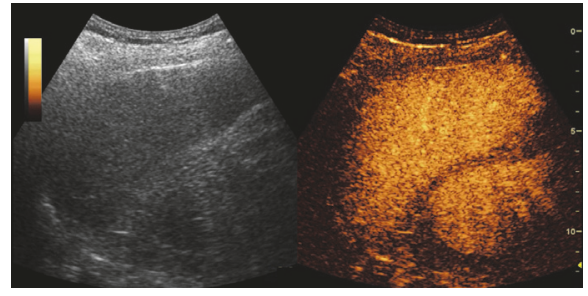

(b)

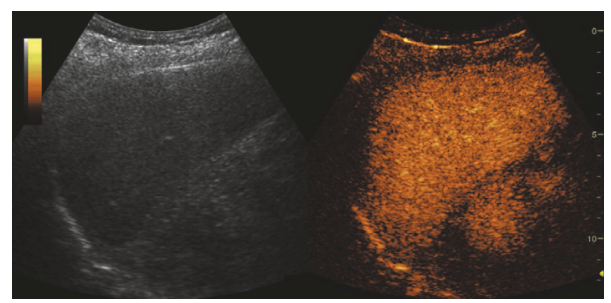

(c)

FiguRE 3: Small recurrent lesion $(15 \mathrm{~mm})$ indistinctive on B mode ultrasound showed homogeneous hyperenhancement during arterial phase (a). Isoenhancement during the portal venous (b) and late phase (c) on contrast-enhanced ultrasound. The arrow in (a) refers to homogeneously hyperenhanced small recurrence lesion during arterial phase. 
only during the arterial phase. During the first CEUS, the primary CEUS feature to be searched for detection of HCCs in cirrhosis is the hyperenhancement in the arterial phase.

Meanwhile the detection of subsequent hypoenhancement during portal or late phase is also requested to definitively establish the diagnosis of HCC $[2,18,19,24]$. The rates of arterial hyperenhancement in HCCs are reported to be increasing with size: in lesions $\leq 2.0 \mathrm{~cm}$ and equal to $3.0 \mathrm{~cm}$, they are between 40 and $70 \%$, respectively [25, 26]. Most of FLLs are smaller than $2 \mathrm{~cm}$ in our current research; as a result, most of HCCs in our current study showed homogeneous and complete hyperenhancement pattern during the arterial phase in liver cirrhosis. The washout tends to start later in HCC $[27,28]$. In our study, $51.3 \%$ of lesions were hypoenhanced after $180 \mathrm{~s}$ after injection of the contrast agent. Therefore, for those indistinctive HCCs on BMUS, it is important to prolong the observation of contrast enhancement in cirrhosis for up to 4 minutes [2].

The presence or absence of liver metastases plays a vital important role in the choice of therapy. Therefore, it is crucial to have accurate preoperative methods for the detection of liver metastases [29]. Despite advances in modern imaging techniques, assessing the presence of liver metastases remains challenging; currently there is no reliable method for detecting small, occult liver metastases [30, 31]. In our study, liver metastases are found on CEUS in $21.4 \%$ of patients which are indistinctive on BMUS. For those metastasis malignant lesions, isoenhancement during arterial phase and early washout during PVLP are more common. Metastases consistently show rapid washout ( $<60$ seconds) [32]. CEUS provides a fast and reliable diagnosis, making any other further imaging investigations unnecessary. The mean diameter of those metastasis lesions is $12 \mathrm{~mm}$; therefore these patients are being early diagnosed and referred for further treatment. According to the up-to-date standard, CEUS should be recommended in the follow-up of patients with colon cancer in addition to BMUS [33].

For recurrent HCC lesions, arterial hyperenhancement and isoenhancement during PVLP are more common. A possible explanation for nonvisualization of recurrent lesions on BMUS images in our study may be the interference of scars from previous operation or minimal invasive treatment (such as radiofrequency ablation and percutaneous ethanol injection) or that neoangiogenic process may not be fully developed in smaller recurrent lesions $[34,35]$.

\section{Conclusion}

In conclusion, 2-step CEUS may provide added diagnostic values in those FLLs appearing indistinctive on BMUS. Presence of early arterial enhancement and washout area during PVLP may be helpful for increasing the detection rate of those lesions.

\section{Conflicts of Interest}

The authors declare that they have no conflicts of interest.

\section{Authors' Contributions}

Professor Wen-Ping Wang is accepting full responsibility for the conduct of the study. He and all others had access to the data. All had control of the decision to publish; Professor Wen-Ping Wang contributed to planning the study; all authors contributed to conducting the study, collecting and/or interpreting data, and critical revision of the manuscript for important intellectual content; Wen-Ping Wang and Dong Yi contributed to drafting the manuscript; Wen-Ping Wang, Feng Mao, and Dong Yi were responsible for statistical analysis. Each author made a statement that they approved the final draft submitted.

\section{Acknowledgments}

This study was supported by the National Natural Science Foundation of China (NSFC Grant 81371577, NSFC Grant 81571676, and NSFC Grant 81501471).

\section{References}

[1] A. Anaye, G. Perrenoud, N. Rognin et al., "Differentiation of focal liver lesions: usefulness of parametric imaging with contrast-enhanced US," Radiology, vol. 261, no. 1, pp. 300-310, 2011.

[2] V. Cantisani, E. David, F. M. Meloni et al., "Recall strategies for patients found to have a nodule in cirrhosis: is there still a role for CEUS?" Medical Ultrasonography, vol. 17, no. 4, pp. 515-520, 2015.

[3] D. Wildner, T. Bernatik, C. Greis, K. Seitz, M. F. Neurath, and D. Strobel, "CEUS in hepatocellular carcinoma and intrahepatic cholangiocellular carcinoma in 320 patients-early or late washout matters: a subanalysis of the DEGUM multicenter trial," Ultraschall in der Medizin, vol. 36, no. 2, pp. 132-139, 2015.

[4] T. V. Bartolotta, A. Taibbi, M. Midiri, D. Matranga, L. Solbiati, and R. Lagalla, "Indeterminate focal liver lesions incidentally discovered at gray-scale US: role of contrast-enhanced sonography," Investigative Radiology, vol. 46, no. 2, pp. 106-115, 2011.

[5] D. Wildner, L. Pfeifer, R. S. Goertz et al., "Dynamic contrastenhanced ultrasound (DCE-US) for the characterization of hepatocellular carcinoma and cholangiocellular carcinoma," Ultraschall in der Medizin, vol. 35, no. 6, pp. 522-527, 2014.

[6] J. Jacob, A. Deganello, M. E. Sellars, N. Hadzic, and P. S. Sidhu, "Contrast enhanced ultrasound (CEUS) characterization of grey-scale sonographic indeterminate focal liver lesions in pediatric practice," Ultraschall in der Medizin, vol. 34, no. 6, pp. 529-540, 2013.

[7] R. Lencioni, C. Della Pina, L. Crocetti, E. Bozzi, and D. Cioni, "Clinical management of focal liver lesions: the key role of real-time contrast-enhanced US," European Radiology, vol. 17, supplement 6, pp. F73-F79, 2007.

[8] A. Oldenburg and T. Albrecht, "Baseline and contrast-enhanced ultrasound of the liver in tumor patients," Ultraschall in der Medizin, vol. 29, no. 5, pp. 488-498, 2008.

[9] P. J. A. Robinson, "Imaging liver metastases: current limitations and future prospects," British Journal of Radiology, vol. 73, no. 867, pp. 234-241, 2000.

[10] V. Catala, C. Nicolau, R. Vilana et al., "Characterization of focal liver lesions: comparative study of contrast-enhanced 
ultrasound versus spiral computed tomography," European Radiology, vol. 17, no. 4, pp. 1066-1073, 2007.

[11] C. F. Dietrich, M. E. Maddalena, X. W. Cui, D. SchreiberDietrich, and A. Ignee, "Liver tumor characterization-review of the literature," Ultraschall in der Medizin, vol. 33, pp. S3-S10, 2012.

[12] C. F. Dietrich, "Comments and illustrations regarding the guidelines and good clinical practice recommendations for contrast-enhanced ultrasound (CEUS) - update 2008," Ultraschall in der Medizin, Supplement, vol. 29, no. 4, pp. S188-S202, 2008.

[13] C. F. Dietrich, "Liver tumor characterization-comments and illustrations regarding guidelines," Ultraschall in der Medizin, vol. 33, pp. S22-S30, 2012.

[14] C. F. Dietrich, X. W. Cui, B. Boozari, M. Hocke, and A. Ignee, "Contrast-enhanced ultrasound (CEUS) in the diagnostic algorithm of hepatocellular and cholangiocellular carcinoma, comments on the AASLD guidelines," Ultraschall in der Medizin, vol. 33, no. 1, pp. S57-S66, 2012.

[15] T. Albrecht, M. J. K. Blomley, P. N. Burns et al., "Improved detection of hepatic metastases with pulse-inversion US during the liver-specific phase of SHU 508A: multicenter study," Radiology, vol. 227, no. 2, pp. 361-370, 2003.

[16] A. Muhi, T. Ichikawa, U. Motosugi et al., "Diagnosis of colorectal hepatic metastases: comparison of contrast-enhanced CT, contrast-enhanced US, superparamagnetic iron oxideenhanced MRI, and gadoxetic acid-enhanced MRI," Journal of Magnetic Resonance Imaging, vol. 34, no. 2, pp. 326-335, 2011.

[17] K. Seitz, D. Strobel, T. Bernatik et al., "Contrast-Enhanced ultrasound (CEUS) for the characterization of focal liver lesions prospective comparison in clinical practice: CEUS vs. CT (DEGUM Multicenter Trial) parts of this manuscript were presented at the ultrasound dreiländertreffen 2008, Davos," Ultraschall in der Medizin, vol. 30, no. 4, pp. 383-389, 2009.

[18] M. Claudon, C. F. Dietrich, B. I. Choi et al., "Guidelines and good clinical practice recommendations for Contrast Enhanced Ultrasound (CEUS) in the liver-update 2012: a WFUMBEFSUMB initiative in cooperation with representatives of AFSUMB, AIUM, ASUM, FLAUS and ICUS," Ultraschall in der Medizin, vol. 34, no. 1, pp. 11-29, 2013.

[19] M. Claudon, C. F. Dietrich, B. I. Choi et al., "Guidelines and good clinical practice recommendations for contrast enhanced ultrasound (CEUS) in the liver-update 2012. A WFUMBEFSUMB initiative in cooperation with representatives of AFSUMB, AIUM, ASUM, FLAUS and ICUS," Ultrasound in Medicine and Biology, vol. 39, no. 2, pp. 187-210, 2013.

[20] Y. Dong, W.-P. Wang, Y.-H. Gan, B.-J. Huang, and H. Ding, "Radiofrequency ablation guided by contrast-enhanced ultrasound for hepatic malignancies: preliminary results," Clinical Radiology, vol. 69, no. 11, pp. 1129-1135, 2014.

[21] L. Chiorean, V. Cantisani, C. Jenssen, P. S. Sidhu, U. Baum, and C. F. Dietrich, "Focal masses in a non-cirrhotic liver: the additional benefit of CEUS over baseline imaging," European Journal of Radiology, vol. 84, no. 9, pp. 1636-1643, 2015.

[22] M. D’Onofrio, S. Crosara, R. De Robertis, S. Canestrini, and R. P. Mucelli, "Contrast-enhanced ultrasound of focal liver lesions," American Journal of Roentgenology, vol. 205, no. 1, pp. W56W66, 2015.

[23] C. F. Dietrich, "Characterisation of focal liver lesions with contrast enhanced ultrasonography," European Journal of Radiology, vol. 51, supplement, pp. S9-S17, 2004.
[24] H.-J. Jang, T. K. Kim, P. N. Burns, and S. R. Wilson, "Enhancement patterns of hepatocellular carcinoma at contrast-enhanced US: comparison with histologic differentiation," Radiology, vol. 244, no. 3, pp. 898-906, 2007.

[25] A. Forner, R. Vilana, C. Ayuso et al., "Diagnosis of hepatic nodules $20 \mathrm{~mm}$ or smaller in cirrhosis: prospective validation of the noninvasive diagnostic criteria for hepatocellular carcinoma," Hepatology, vol. 47, no. 1, pp. 97-104, 2008.

[26] A. Sangiovanni, M. A. Manini, M. Iavarone et al., "The diagnostic and economic impact of contrast imaging techniques in the diagnosis of small hepatocellular carcinoma in cirrhosis," Gut, vol. 59, no. 5, pp. 638-644, 2010.

[27] L. Bolondi, S. Gaiani, N. Celli et al., "Characterization of small nodules in cirrhosis by assessment of vascularity: the problem of hypovascular hepatocellular carcinoma," Hepatology, vol. 42, no. 1, pp. 27-34, 2005.

[28] S. Leoni, F. Piscaglia, R. Golfieri et al., “The impact of vascular and nonvascular findings on the noninvasive diagnosis of small hepatocellular carcinoma based on the EASL and AASLD criteria," American Journal of Gastroenterology, vol. 105, no. 3, pp. 599-609, 2010.

[29] P. Taimr, V. L. Jongerius, C. J. Pek et al., "Liver contrastenhanced ultrasound improves detection of liver metastases in patients with pancreatic or periampullary cancer," Ultrasound in Medicine and Biology, vol. 41, no. 12, pp. 3063-3069, 2015.

[30] K. Holzapfel, C. Reiser-Erkan, A. A. Fingerle et al., "Comparison of diffusion-weighted MR imaging and multidetector-row CT in the detection of liver metastases in patients operated for pancreatic cancer," Abdominal Imaging, vol. 36, no. 2, pp. 179184, 2011.

[31] M. Kopljar, L. Patrlj, Z. Busic et al., "Potential use of Doppler perfusion index in detection of occult liver metastases from colorectal cancer," Hepatobiliary Surgery and Nutrition, vol. 3, no. 5, pp. 259-267, 2014.

[32] D. Bhayana, T. K. Kim, H.-J. Jang, P. N. Burns, and S. R. Wilson, "Hypervascular liver masses on contrast-enhanced ultrasound: the importance of washout," American Journal of Roentgenology, vol. 194, no. 4, pp. 977-983, 2010.

[33] T. Bernatik, A. Schuler, G. Kunze et al., "Benefit of ContrastEnhanced Ultrasound (CEUS) in the follow-up care of patients with colon cancer: a prospective multicenter study," Ultraschall in der Medizin, vol. 36, no. 6, pp. 590-593, 2015.

[34] S. Leoni, F. Piscaglia, A. Granito et al., "Characterization of primary and recurrent nodules in liver cirrhosis using contrast-enhanced ultrasound: which vascular criteria should be adopted?" Ultraschall in der Medizin, vol. 34, no. 3, pp. 280 287, 2013.

[35] Y. Wang, J. Liao, W. Qi, L. Xie, and Y. Li, "Predictive value of conventional ultrasound and contrast-enhanced ultrasound in early recurrence of hepatocellular carcinoma after surgical resection," Ultrasound in Medicine and Biology, vol. 42, no. 5, pp. 1042-1048, 2016. 


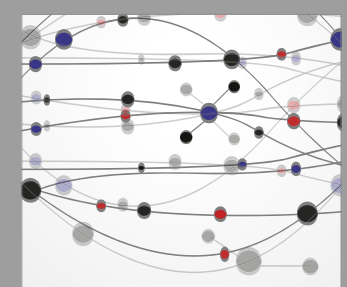

The Scientific World Journal
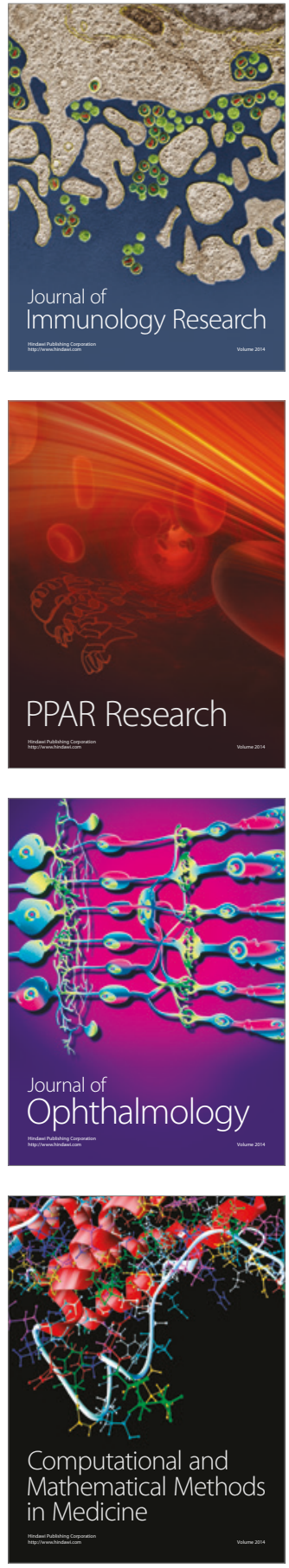

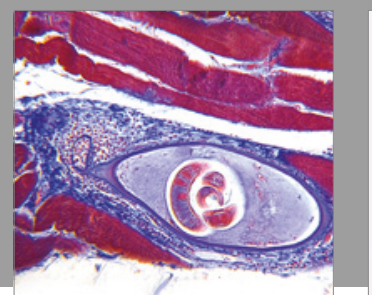

Gastroenterology Research and Practice
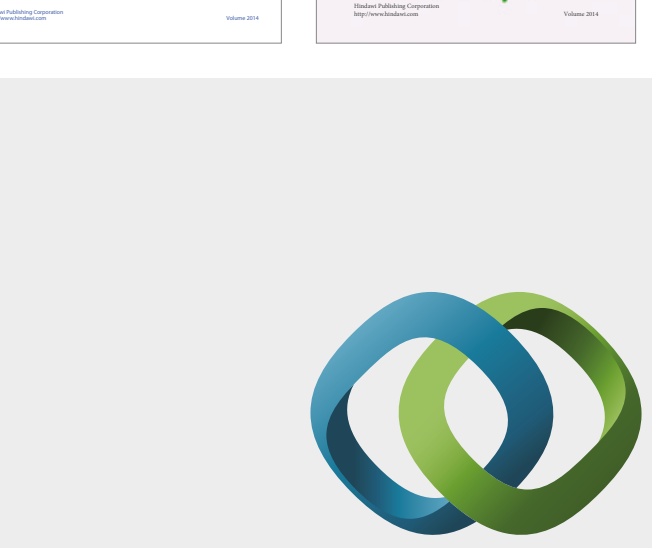

\section{Hindawi}

Submit your manuscripts at

https://www.hindawi.com
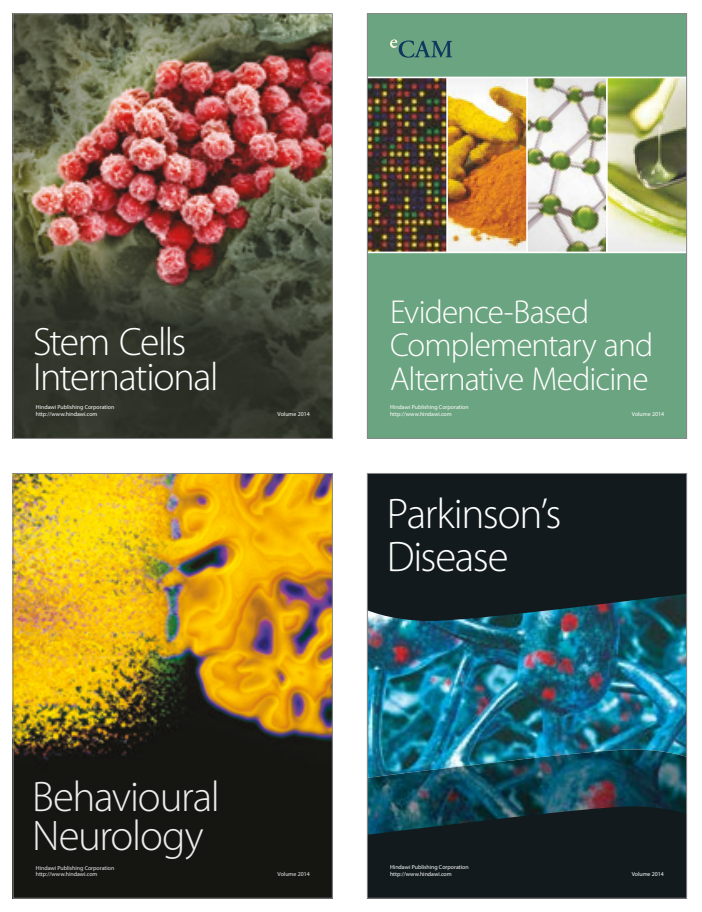
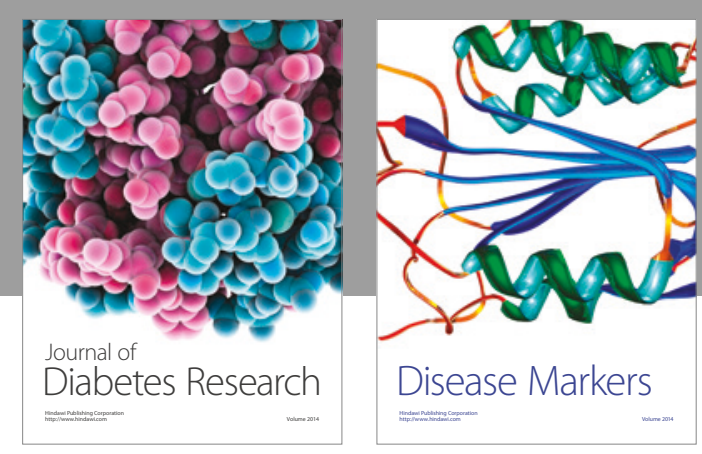

Disease Markers
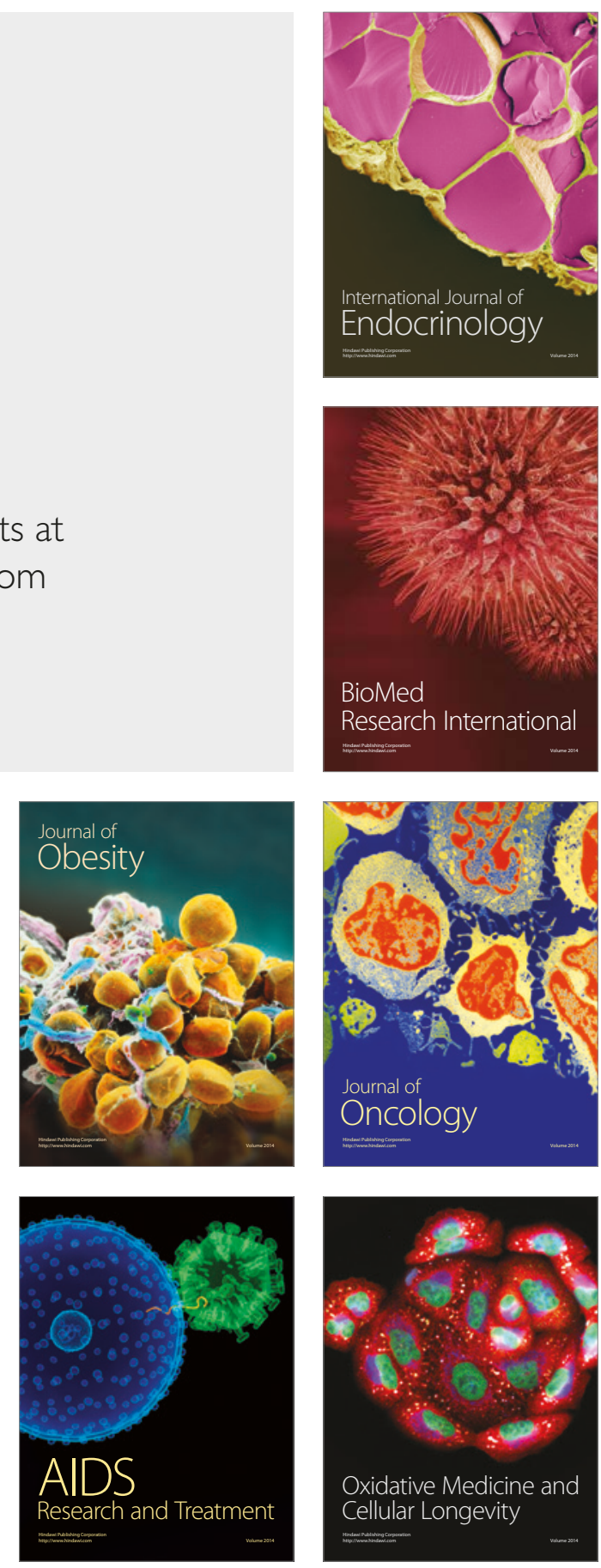